\title{
Factors that influence the levels of cerebrospinal fluid biomarkers in memory clinic patients
}

\author{
Anne-Brita Knapskog $^{1 *}$ D, Rannveig Sakshaug Eldholm², Anne Braekhus ${ }^{1}$, Knut Engedal ${ }^{3}$ and Ingvild Saltvedt ${ }^{2,4}$
}

\begin{abstract}
Background: The cerebrospinal fluid (CSF) biomarkers amyloid $\beta$ (A $\beta$ ), phospho tau (P-tau) and total tau (T-tau) are used increasingly to support a clinical diagnosis of Alzheimer's disease. The diagnostic power of these biomarkers has been reported to vary among different studies' results. The results are poorer when heterogeneous groups of patients have been included compared to studies where patients with Alzheimer's dementia (AD) and healthy controls have been studied. The aim of this study was to examine if age, APOE genotype and sex were associated with the levels of CSF biomarkers among patients referred to a memory clinic.
\end{abstract}

Methods: We included 257 patients from two memory clinics who had been assessed for dementia, including lumbar puncture.

Results: The mean age of the patients was 68.1 (SD: 8.0) years; $50.2 \%$ were women and 66.5\% were APOE $\varepsilon 4$ positive. Of these patients, $80.5 \%$ were diagnosed with AD or amnestic MCl. Both APOE $\varepsilon 4$ and increasing age were associated with decreasing levels of $A \beta$, but not the levels of the tau proteins. In multiple regression analyses, disease stage, defined as a MMSE $\geq 25$ or $<25$, influenced factors associated with the CSF biomarkers. Among those with MMSE score $\geq 25$, age, APOE $\varepsilon 4$ genotype, and MMSE score, in addition to a diagnosis of AD, were associated with $A \beta$ level, with an explained variance of 0.43 . When using P-tau or T-tau as a dependent variable, the presence of one or two APOE $\varepsilon 4$ alleles, and MMSE score influenced the results, in addition to the diagnosis of AD. The explained variance was lower for P-tau (0.26) and for T-tau (0.32). Among those with MMSE $<25$, these variables explained very little of the variance. There were no gender differences.

Conclusions: We found that factors in addition to a diagnosis of AD, were associated with the levels of CSF biomarkers. Among those with MMSE $\geq 25$, lower levels of $A \beta$ were associated with several factors including increasing age. This is not reflected in clinical practice, where age-specific cutoffs exist only for T-tau. In this study, age was not associated with the levels of tau proteins.

Keywords: Cerebrospinal fluid biomarkers, Alzheimer's Dementia, Mild cognitive impairment, Memory clinic

\section{Background}

Alzheimer's dementia (AD) is the most common cause of dementia, with an increasing incidence as the world's population is getting older [1, 2]. Approximately 47 million people suffer from dementia worldwide, and there are around 10 million new cases every year. AD contributes to 60 to $70 \%$ of these cases [3]. Several pathological changes are seen in the brains of patients

\footnotetext{
* Correspondence: anne-brita@knapskog.net

1 Department of Geriatric Medicine, The memory clinic, Oslo University

Hospital, Ullevaal, Postboks 4956, Nydalen, 0424 Oslo, Norway

Full list of author information is available at the end of the article
}

with $\mathrm{AD}$, but none of these changes have, as yet, proven to be specific markers of the disease. AD is still a clinical diagnosis according to various criteria, including the NINCDS-ADRDA criteria [4]. Complemental examinations such as magnetic resonance imaging brain scans (MRI) or positron emission tomography (PET) and cerebrospinal fluid (CSF) biomarkers are being performed increasingly for verification of the diagnosis.

According to the amyloid cascade hypothesis, the disease starts with the deposition of extracellular amyloid plaques, followed by phosphorylation of the tau protein 
within the neurons, which results in the formation of neurofibrillary tangles and cell loss [5, 6]. Additionally, vascular changes, inflammation, and oxidative stress reactions are seen [7]. The amount of CSF amyloid $\beta(A \beta)$ is considered to inversely reflect the amyloid burden in the brain. However, a decreased level of A $\beta$ in the CSF is seen not only in AD, but also with increasing age and in patients with dementia with Lewy Bodies (DLB) and vascular dementia $(\mathrm{VaD})[8]$.

The increasing deposition of amyloid in the brain with advancing age is observed not only in people with cognitive impairment but also in individuals with normal cognition [9]. Among people in their 50s and 60s, hardly any amyloidosis is found, while by 80 to 90 years of age, nearly half of all healthy people have both amyloidosis and neurodegenerative changes (such as hippocampal atrophy) in the brain, as verified by MRI and PET [10].

Furthermore, increased levels of total tau (T-tau) and phosphorylated tau (P-tau) in the neurons in the brain result in increased levels of T-tau and P-tau in the CSF of typical patients diagnosed with $\mathrm{AD}$ [11]. It has been suggested that both the changes in tau and $A \beta$ in the CSF may precede clinical symptoms by decades $[12,13]$.

Apolipoprotein E (APOE) is an important transporter of cholesterol in the brain. It also plays a significant role in amyloid metabolism and in the clearance of amyloid $[14,15]$. There are three isoforms of $\operatorname{APOE}(\varepsilon 2, \varepsilon 3$, and $\varepsilon 4)$, and APOE $\varepsilon 4$ has the poorest capacity for transporting cholesterol and clearing $A \beta$ in the brain [16]. In people who are APOE $\varepsilon 4$ positive, more amyloidosis is found $[9,10]$, meaning there is an additive effect of age and APOE \&4 [17]. This is found in postmortem studies of cognitively intact older people as well [13]. Several studies have shown lower levels of CSF A $\beta$ in APOE $\varepsilon 4$-positive people both with and without dementia, and there seems to be a genetic dose-dependent correlation with the lowest levels in the homozygote group $[18,19]$.

In addition, $\mathrm{APOE} \varepsilon 4$ has $\mathrm{A} \beta$ independent neurotoxic effects including formation of neurotoxic fragments caused by deviant proteolysis of APOE $\varepsilon 4$ and, thereby, increased phosphorylation of tau and impaired mitochondrial function [15].

$\mathrm{APOE} \varepsilon 4$ is the strongest known genetic risk factor for sporadic AD [20, 21]. Worldwide, approximately $15 \%$ of all people have one or two alleles of APOE $\varepsilon 4$, but the prevalence is found to be higher in Northern Europe [22]. The majority of APOE $\varepsilon 4$ carriers are heterozygote $(2 / 4$ or $3 / 4)$ carriers, and only a small percentage are homozygote $(4 / 4)$ carriers. People who are heterozygote carriers for APOE $\varepsilon 4$ have a $20 \%$ to $30 \%$ lifetime risk of developing $\mathrm{AD}$, whereas homozygote carriers have a lifetime risk of approximately $50 \%$ [23]. This risk of developing $\mathrm{AD}$ associated with $\mathrm{APOE} \varepsilon 4$ is higher for women than for men [23].
Over the last 20 years, there has been a considerable amount of research focused on the diagnostic power of CSF biomarkers. Comparing patients with AD to healthy controls, CSF biomarkers are shown to differentiate healthy persons from those who have the disease very accurately, with reported sensitivity (SS) of $85-94 \%$, and specificity (SP) of $83-100 \%$ [24]. Comparing patients with $\mathrm{AD}$ to patients with other dementia disorders, the results are less convincing, with lower SS and SP [25]. When keeping SS at a level of $85 \%$, an SP between $39 \%$ and $90 \%$ is reported in various studies [26]. Thus, the aim of the present study is to examine the influence of factors other than a diagnosis of $\mathrm{AD}$ on the levels of CSF biomarkers among a heterogeneous sample of patients referred to a memory clinic.

\section{Methods}

Aim

Based on the literature, we suggest that age, sex, and APOE genotype may influence the levels of CSF biomarkers of $\mathrm{AD}$ in a significant way, especially for the level of $A \beta$ in the CSF.

\section{Design}

The study is a cross-sectional study performed among patients referred to two university memory clinics for diagnostic assessment of dementia in Norway.

\section{Participants}

We included data from the first visits of 257 patients referred from their primary care doctors to the memory clinics at Oslo University Hospital (OUH), Ullevaal (156 patients), and St. Olav University Hospital (97 patients), Trondheim, in the period from October 2009 to January 2015. The only inclusion criteria for participation were that the patient, in addition to the clinical examination, had undergone lumbar puncture for measurement of $A \beta$ and tau proteins, and that the APOE genotype was known. There were no exclusion criteria.

\section{Examination and procedures for diagnoses}

All patients were examined in a standardized and comprehensive manner according to a research protocol [27]. Clinical information was obtained from the patients, their caregivers, their general practitioners, and hospital records. The patients were examined by trained physicians and underwent a neuropsychological examination (including, among others, the Mini-Mental State Examination (MMSE), the Consortium to Establish a Registry of Alzheimer's Disease [CERAD] 10-item word list, the Clock Drawing Test [CDT], and the Trail Making Tests A and B [TMT A and B]). In addition, physical examinations including blood and spinal fluid samples and magnetic resonance imaging brain scans 
(MRI) were performed. In some cases, single-photon emission computer tomography (SPECT), DaTscan (Ioflupane I 123 injection), or ${ }^{18} \mathrm{~F}$-2-fluoro-2-deoxy-Dglucose positron emission tomography (FDG-PET) was performed when indicated in order to reach a specific diagnosis.

\section{Spinal fluid biomarkers}

The two hospitals performed lumbar punctures and collection of spinal fluid according to an identical procedure. The lumbar punctures were carried out between 9 a.m. and 11 a.m. Spinal fluid was collected in cryotubes and, within thirty minutes of collection, centrifuged for ten minutes at $2000 \mathrm{G}$. All the samples were analyzed at the same laboratory at Akershus University Hospital (AHUS). Samples were either sent to the laboratory on the same day or frozen at -20 degrees Celsius and later sent in a frozen state. All samples were analyzed with the Innotest kit (Innogenetics, Ghent, Belgium) for all three biomarkers. The laboratory follows its own internal quality control program by using control samples in every batch of samples analyzed. It is also part of the Alzheimer's Association QC program for CSF biomarkers [28]. The Clinical Neurochemistry Laboratory in Gothenburg, Sweden, is in charge of the program in collaboration with the Alzheimer's Association. The laboratory at AHUS receives samples from this program for analysis three times a year. The cutoffs for the three biomarkers recommended from the laboratory were applied: amyloid- $\beta\left(A \beta_{42}\right):>550$, phospho tau (P-tau): $<80$, and total tau (T-tau): < 300 for patients under 50 years, $<450$ for patients ages 50 to 69 , and $<500$ for those 70 years and older. Age-specific cut offs exist for total tau only.

\section{APOE genotyping}

APOE genotyping was performed using the Illumina Infinium OmniExpress v1.1 chip at deCODE Genetics, Reykjavik, Iceland for all included patients.

\section{Diagnostic criteria and diagnoses}

All patients included in this study had a cognitive impairment. For the diagnosis of mild cognitive impairment (MCI), the Winblad criteria were used [29]. The $\mathrm{MCI}$ group was further divided into two groups, amnestic MCI and non-amnestic MCI. Amnestic MCI was defined as patients with MCI with memory complaints as expressed by the patient and/or caregiver and a score equivalent to or below -1.5 SD on the CERAD 10-item delayed recall test, with no other obvious aetiology for cognitive impairment, including MRI findings.

The ICD-10 criteria for research were used to diagnose the patients with dementia. Diagnoses of Alzheimer's dementia ( $\mathrm{AD})$, vascular dementia $(\mathrm{VaD})$, and dementia related to Parkinson's disease (PDD) were made according to the ICD-10 criteria for research as well [30]. The Manchester-Lund criteria [31] were applied for frontotemporal dementia (FTD) and for dementia with Lewy Bodies (DLB); the consensus criteria according to McKeith et al. [32] were used.

\section{Statistics}

The statistics were carried out using IBM SPSS, version 22. Descriptive analyses of the whole sample and the subgroups $\mathrm{MCI}, \mathrm{AD}$, and other dementias were performed (mean, SD, \%). As the main aim of the study was to examine factors that could influence the ability of CSF markers to differentiate AD from other disorders, in the further analyses we dichotomized the patients into two groups according to their clinical diagnoses, 207 patients with $\mathrm{AD}$ or amnestic $\mathrm{MCI}$ and 50 patients with other diagnoses (other MCI, and other forms of dementia). In the multiple linear regression analyses, unadjusted and adjusted analyses were carried out with the three spinal fluid biomarkers as dependent variables. As independent variables, we included age, sex, diagnoses, APOE genotype, and cognitive tests (MMSE and TMT B). Before conducting the adjusted regression analyses, we checked for intercorrelations between the independent variables using the Spearman correlation coefficient. Variables that intercorrelated with a value higher than 0.6 were not included in the analyses. Thus, the CDT, CERAD's 10-item word list and TMT A had to be excluded from further analyses. The correlation between T-tau and P-tau was high (Spearman's rho 0.91) but low between $\mathrm{A} \beta$ and T-tau or P-tau (Spearman's rho -0.33 and -0.3 , respectively). We also checked for interaction between disease stage (measured by MMSE) and the other predictors included in the regression analyses, and found that the association of the predictors on the biomarkers was dependent on disease stage. In the further analyses we decided to divide the patients into two groups, patients with a MMSE score $\geq 25$ and patients with a MMSE score $<25$. Multiple regression analyses were performed using both standard and stepwise (enter and backward) methods. The results were the same, and the results using the backward method will be presented. Independent variables with $P$ values $<0.2$ were included in the adjusted analyses.

\section{Results}

\section{Characteristics of the patients}

Of the 257 patients included, 129 (50.2\%) were women. The mean age for the whole group was 68.1 years (SD: 8.0; range 43-84 years) with no difference in age between women and men (68.0 [SD: 7.1] vs. 68.2 [SD: 8.6] years respectively, $p=0.86$ ). The mean years of education were 12.4 (SD: 3.6); and the mean MMSE 
score was 23.9 (SD: 4.4). See Table 1 for further details regarding the characteristics of the subgroups of the patients.

The patients recruited from St. Olav University Hospital differed from the patients at OUH in regard to age, education, and cognitive impairment. They were older (mean 72.8 years [SD: 4.6] vs. 65.2 years [SD: 8.2] $p<0.001$ ); they had a lower level of education (mean 10.4 years [SD: 2.9] vs. 13.6 years [SD: 3.5$] p<0.001$ ); and they had significantly lower scores on the MMSE (median 23.0 vs. $26.0, p=0.001$ ) and on the delayedrecall test of the CERAD's 10-item word list (mean 1.7 words [SD: 1.9] vs. 2.3 words, [SD 2.2] $p=0.05$ ).

There was a significant difference between the patient group with a MMSE score $<25$ compared with the group with a MMSE score $\geq 25$. In the group with MMSE scores <25, mean age was higher (69.4 [SD: 7.2] vs.67.1 years $[8.5] p=0.02)$; there were more women (61.3\% vs. $41.0 \%, p=0.001)$; the diagnosis of $\mathrm{AD} / \mathrm{aMCI}$ was more frequent $(89.9 \%$ vs. $73.1 \%, p=0.001)$. In addition; the CSF biomarkers were more pathological (A $\beta$ mean 571.5 [SD: 200.9] vs. 729.8 [SD: 296.2] $p<0.001$, P-tau mean 80.3 [SD: 34.9] vs. 70.7 [SD: 31.8] $p=0.03$, and T-tau mean 693.6 [SD: 358.7] vs. 545.5 [SD: 334.9] $\mathrm{p}<0.001)$.

\section{Diagnoses}

Of the whole group of 257 patients, 67 (26.1\%) patients were diagnosed with MCI; 165 (64.2\%) had AD (including 27 [10.5\%] patients with mixed $\mathrm{AD} / \mathrm{VaD}$ ) with typical $\mathrm{AD}$ symptomatology. Of the remaining 25 (9.7\%) patients, 1
(0.4\%) had VaD; 3 (1.2\%) had FTD; 14 (5.5\%) were diagnosed with PDD/DLB; and 7 (2.8\%) had other dementias. Of the 67 patients with MCI, 42 (62.7\%) were classified as patients having amnestic MCI. The remaining 25 (37.3\%) patients met the criteria for MCI due to cerebrovascular disease or other physical disorders, psychiatric disorders such as depression, or drug and/or alcohol abuse.

\section{Associations between the levels of $A \beta$, diagnosis, and APOE genotype in different age groups}

Of the whole study population, $66.5 \%$ were APOE $\varepsilon 4$ carriers; $48.2 \%$ were heterozygote; and $18.3 \%$ were homozygote. The prevalence of none, one, or two APOE $\varepsilon 4$ alleles differed in the three different age groups. In the youngest group (below 65 years of age), approximately $58 \%$ were heterozygote or homozygote, whereas in the groups older than 65 years, approximately two-thirds were APOE $\varepsilon 4$ carriers. There was no gender difference in the prevalence of the APOE $\varepsilon 4$ allele ( $p$ value 0.29 ) or between patients with $\mathrm{AD}$ and patients with amnestic MCI $(71.5 \%$ vs. $66.7 \%$ positive, $p=0.54$ ). Among patients with $\mathrm{AD}$ and amnestic $\mathrm{MCI}$, the level of $\mathrm{A} \beta$ was significant lower in APOE $\varepsilon 4$ carriers in the two oldest age groups. There was an age-dependent decrease in the CSF A $\beta$ in non-carriers and in patients with one $\varepsilon 4$ allele, while this was not statistically significant in homozygote patients. See Table 2 for further details. A similar age-dependent increase in the CSF P-tau or T-tau was not shown in any of the groups. However, in the

Table 1 Characteristics of the patients

\begin{tabular}{|c|c|c|c|c|}
\hline & MCl patients $(N=67)$ & AD patients $(N=165)$ & Other dementias $(N=25)$ & $P$ \\
\hline \multicolumn{5}{|l|}{ Patient characteristics } \\
\hline Age [mean (SD)] & $66.7(9.3)$ & $68.7(7.1)$ & $67.8(9.3)$ & $0.22^{\mathrm{a}}$ \\
\hline Women [n (\%)] & $34(50.7)$ & $87(52.7)$ & $8(32.0)$ & $0.15^{b}$ \\
\hline Education [mean (SD)] & $12.8(4.0)$ & $12.2(3.4)$ & $12.3(3.9)$ & $0.54^{\mathrm{a}}$ \\
\hline ApoE $\varepsilon 4$ positive [n (\%)] & $40(59.7)$ & $118(71.5)$ & $13(52.0)$ & $0.06^{\mathrm{b}}$ \\
\hline \multicolumn{5}{|l|}{ Cognition } \\
\hline MMSE [mean (SD)] & $26.9(3.0)$ & $22.7(4.4)$ & $24.2(3.8)$ & $<0.001^{\mathrm{C}}$ \\
\hline CDT accepted [n (\%)] & $54(83.1)$ & $76(47.5)$ & $15(60.0)$ & $<0.001^{\mathrm{b}}$ \\
\hline TMT A $\geq-2$ SD [n (\%)] & $55(83.3)$ & $89(58.0)$ & $10(41.7)$ & $<0.001^{\mathrm{b}}$ \\
\hline TMT $\mathrm{B} \geq-2 \mathrm{SD}[\mathrm{n}(\%)]$ & $45(69.2)$ & $57(41.0)$ & $7(31.8)$ & $<0.001^{\mathrm{b}}$ \\
\hline \multicolumn{5}{|l|}{ CSF biomarkers } \\
\hline$A \beta[$ mean (SD)] & 799.1 (315.6) & $583.3(210.5)$ & $735.9(281.7)$ & $<0.001^{\mathrm{C}}$ \\
\hline T-Tau [mean (SD)] & $464.7(260.8)$ & $717.0(357.9)$ & $305.1(165.4)$ & $<0.001^{\mathrm{C}}$ \\
\hline P-tau [mean (SD)] & $63.6(24.4)$ & $83.6(35.0)$ & $47.3(16.7)$ & $<0.001^{\mathrm{C}}$ \\
\hline
\end{tabular}

$M C l$ mild cognitive impairment, $A D$ Alzheimer's Disease, MMSE Mini Mental State Examination, CDT the Clock Drawing Test, TMT Trail Making Test A and B, $A \beta$ Amyloid $\beta, T$-tau total tau, $P$-tau phosho tau

$\%=$ valid percent without missing

${ }^{a}$ one-way ANOVA, ${ }^{b}$ Chi square, ${ }^{C}$ Kruskal-Wallis test 
Table 2 Associations between the levels of A $\beta$, diagnosis, and ApoE genotype in different age groups

\begin{tabular}{|c|c|c|c|c|c|c|c|}
\hline \multirow[b]{3}{*}{ ApoE $\varepsilon 4$} & \multicolumn{7}{|c|}{ Alzheimer's Dementia/amnestic mild cognitive impairment } \\
\hline & \multicolumn{2}{|c|}{ Age $<65$ years $(N=48)$} & \multicolumn{2}{|c|}{ Age $65-74$ years $(N=110)$} & \multicolumn{3}{|c|}{ Age 75 years and older $(N=49)$} \\
\hline & N (\%) & $A \beta[$ mean $(S D)]$ & N (\%) & $A \beta[$ mean $(S D)]$ & N (\%) & $A \beta[$ mean $(S D)]$ & $p$ \\
\hline None & $20(41.7)$ & $774.1(366.0)$ & $23(20.9)$ & $687.4(285.7)$ & $18(36.7)$ & $637.4(264.4)$ & $0.02^{\mathrm{a}}$ \\
\hline Heterozygote & $17(35.4)$ & $659.1(238.3)$ & $61(55.5)$ & $596.9(173.8)$ & $24(49.0)$ & $528.8(123.8)$ & $0.01^{\mathrm{a}}$ \\
\hline Homozygote & $11(22.9)$ & $547.2(113.0)$ & $26(23.6)$ & $498.9(99.9)$ & $7(14.3)$ & $433.9(116.1)$ & $0.37^{\mathrm{a}}$ \\
\hline$P$ & & $\mathrm{p}=0.21^{\mathrm{a}}$ & & $p=0.01^{\mathrm{a}}$ & & $p=0.07 a$ & \\
\hline
\end{tabular}

$A \beta$ amyloid- $\beta$

${ }^{a}$ Kruskal-Wallis test

group 65-74 years the levels of P-tau and T-tau increased with the highest levels in homozygote carriers ( $p$ values 0.009 and 0.004 , respectively).

\section{Multiple regression}

For all three CSF biomarkers, multiple regression analyses gave different results for patients with MMSE $<25$ as compared to patients with MMSE $\geq 25$. Among patients with a MMSE score $\geq 25$, most of the variance for $A \beta$ could be explained by other variables than the diagnosis of $\mathrm{AD}$. For P-tau and T-tau, more of the variance was explained by the diagnosis of AD; see Tables 3, 4 and 5.

In the unadjusted linear regression analyses using $A \beta$ as the dependent variable, all the included variables (diagnosis, age, sex, APOE genotype, and MMSE) were significantly associated with $A \beta$ among patients with a MMSE score $\geq 25$. In the adjusted analyses, all these variables, except sex, remained associated. The diagnosis of $\mathrm{AD}$, age, the presence of one or two APOE $\varepsilon 4$ alleles, and the MMSE score were associated with $A \beta$ with an explained variance of 0.43 .
When using P-tau or T-tau as a dependent variable, the diagnosis of $\mathrm{AD}$, the presence of one or two APOE $\varepsilon 4$ alleles, and the MMSE score were significant in the adjusted analyses. The explained variances for P-tau and T-tau were lower than for $A \beta$ ( 0.26 and 0.32 respectively). See Tables 3, 4 and 5 .

In patients with a MMSE score < 25, approximately $90 \%$ of the patients had a diagnosis of $\mathrm{AD} / \mathrm{aMCI}$, thus diagnoses had to be excluded from the analyses. In the adjusted linear regression analyses using $A \beta$ as the dependent variable, none of the variables in the analyses were associated. Using P-tau or T-tau as the dependent variable, the level of $A \beta$ was the only significant variable. The explained variance was 0.05 for P-tau and 0.07 for T-tau.

\section{Discussion}

In the present descriptive study of memory clinic patients, we studied the association of the CSF biomarkers with other factors than a diagnosis of $\mathrm{AD}$. We found that, in addition to a diagnosis of $\mathrm{AD}$, the age of the patients, the presence of one or two APOE $\varepsilon 4$ alleles, and

Table 3 Multiple linear regression - Amyloid $\beta$

\begin{tabular}{|c|c|c|c|c|c|}
\hline Variables & Unadjusted standardized beta & $P$ & R square & Adjusted standardzied beta & $P$ \\
\hline \multicolumn{6}{|l|}{ Patients with MMSE sum score $\geq 25, N=134$} \\
\hline Diagnoses (AD/aMCl = 2, others $=1)$ & -0.33 & $<0.001$ & 0.1 & -0.18 & 0.02 \\
\hline Age & -0.41 & $<0.001$ & 0.16 & -0.19 & 0.02 \\
\hline $\operatorname{Sex}(1=$ women, 2 = men $)$ & 0.25 & 0.007 & 0.05 & 0.13 & 0.07 \\
\hline ApoE $\varepsilon 4$ genotype $($ neg $=1$, pos $=2)$ & -0.53 & $<0.001$ & 0.27 & -0.41 & $<0.001$ \\
\hline MMSE & 0.34 & $<0.001$ & 0.1 & 0.21 & 0.004 \\
\hline R square & & & & 0.43 & \\
\hline \multicolumn{6}{|l|}{ Patients with MMSE sum score $<25, N=119$} \\
\hline Age & -0.14 & 0.13 & 0.01 & & \\
\hline $\operatorname{Sex}(1=$ women, 2 = men $)$ & -0.09 & 0.34 & -0.001 & & \\
\hline ApoE $\varepsilon 4$ genotype $($ neg $=1$, pos $=2)$ & -0.12 & 0.19 & 0.006 & & \\
\hline MMSE & 0.14 & 0.13 & 0.01 & & \\
\hline R square & & & & 0.0 & \\
\hline
\end{tabular}

AD/aMCl Alzheimer's dementia/amnestic mild cognitive impairment MMSE missing in 4 patients 
Table 4 Multiple linear regression - phospho tau

\begin{tabular}{|c|c|c|c|c|c|}
\hline Variables & Unadjusted standardized beta & $P$ & R square & Adjusted standardized beta & $P$ \\
\hline \multicolumn{6}{|c|}{ Patients with MMSE sum score $\geq 25, N=134$} \\
\hline Diagnoses $(\mathrm{AD} / \mathrm{aMCl}=2$, others $=1)$ & 0.42 & $<0.001$ & 0.17 & 0.36 & $<0.001$ \\
\hline Age & 0.11 & 0.2 & 0.005 & & \\
\hline $\operatorname{Sex}(1=$ women, 2 = men $)$ & -0.18 & 0.04 & 0.03 & & \\
\hline ApoE $\varepsilon 4$ genotype $($ neg $=1$, pos $=2)$ & 0.23 & 0.007 & 0.05 & 0.17 & 0.03 \\
\hline Amyloid $\beta$ & -0.35 & $<0.001$ & 0.11 & & \\
\hline MMSE & -0.33 & $<0.001$ & 0.1 & -0.25 & 0.002 \\
\hline R square, adjusted & & & & 0.26 & \\
\hline \multicolumn{6}{|l|}{ Patients with MMSE sum score $<25, N=119$} \\
\hline Age & 0.12 & 0.18 & 0.007 & & \\
\hline Sex $(1=$ women, 2 = men $)$ & 0.006 & 0.95 & -0.009 & & \\
\hline ApoE $\varepsilon 4$ genotype $($ neg $=1$, pos $=2)$ & 0.17 & 0.07 & 0.02 & & \\
\hline Amyloid $\beta$ & -024 & 0.01 & 0.05 & -0.24 & 0.01 \\
\hline MMSE & -017 & 0.06 & 0.02 & & \\
\hline R square, adjusted & & & & 0.05 & \\
\hline
\end{tabular}

AD/aMCI Alzheimer's dementia/amnestic mild cognitive impairment

MMSE missing in 4 patients

the results on the MMSE were associated with the level of $A \beta$ in the CSF. As for P-tau and T-tau, a diagnosis of $\mathrm{AD}$, the presence of one or two APOE $\varepsilon 4$ alleles, and the MMSE score were associated with the levels of both biomarkers. There were no gender differences. These associations were however, only significant among those with less advanced disease (MMSE $\geq 25$ ). Among the patients with MMSE $<25$, the predictors included in the analyses explained none or very little of the variance.
In this study, $66.5 \%$ of the patients were APOE $\varepsilon 4$ carriers, a prevalence that indicated a selected group of patients compared with the general population [22]. The same frequency is found among patients diagnosed with $\mathrm{AD}$ in Norway earlier [33]. Analysing the whole group of patients, fewer younger patients were APOE $\varepsilon 4$ carriers as compared to the oldest patients of the sample, where two-thirds had at least one APOE \&4 allele. Among patients with $\mathrm{AD} / \mathrm{aMCI}$ we found an APOE $\varepsilon 4$ dose-

Table 5 Multiple linear regression - total tau

\begin{tabular}{|c|c|c|c|c|c|}
\hline Variables & Unadjusted standardized beta & $P$ & R square & Adjusted standardized beta & $P$ \\
\hline \multicolumn{6}{|l|}{ Patients with MMSE sum score $\geq 25, N=134$} \\
\hline Diagnoses $(\mathrm{AD} / \mathrm{aMCl}=2$, others $=1)$ & 0.44 & $<0.001$ & 0.19 & 0.36 & $<0.001$ \\
\hline Age & 0.12 & 0.16 & 0.007 & & \\
\hline $\operatorname{Sex}(1=$ women, 2 = men $)$ & -0.17 & 0.05 & 0.02 & & \\
\hline ApoE $\varepsilon 4$ genotype $($ neg $=1$, pos $=2)$ & 0.26 & 0.002 & 0.06 & 0.2 & 0.008 \\
\hline Amyloid $\beta$ & -0.36 & $<0.001$ & 0.12 & & \\
\hline MMSE & -0.39 & $<0.001$ & 0.15 & -0.31 & 0.001 \\
\hline R square & & & & 0.32 & \\
\hline \multicolumn{6}{|l|}{ Patients with MMSE sum score $<25, N=119$} \\
\hline Age & 0.07 & 0.46 & -0.004 & & \\
\hline Sex $(1=$ women, 2 = men $)$ & -0.02 & 0.87 & -0.008 & & \\
\hline ApoE $\varepsilon 4$ genotype $($ neg $=1$, pos $=2)$ & 0.18 & 0.05 & 0.03 & & \\
\hline Amyloid $\beta$ & -0.25 & 0.005 & 0.06 & -0.24 & 0.01 \\
\hline MMSE & -0.16 & 0.09 & 0.02 & & \\
\hline R square & & & & 0.07 & \\
\hline
\end{tabular}


dependent reduction in $A \beta$ in the CSF among the two oldest age groups (65 years and older), with the lowest level of $A \beta$ among homozygote carriers. There was no association between the APOE genotype and decreased levels of $A \beta$ among the youngest patients (below 65 years of age); see Table 2 . The APOE genotype did not play a significant role in the levels of T-tau and P-tau. The association between APOE $\varepsilon 4$ status and $A \beta$, and the lack of associations between APOE $\varepsilon 4$ status and the tau proteins, was also reported by Mehrabian et al. in patients with $\mathrm{AD}$. In that study, an association between APOE genotype and CSF $A \beta$ was shown among those with late onset $\mathrm{AD}$ (LOAD), but not in early onset $\mathrm{AD}$ (EOAD), [20]. The effect of APOE $\varepsilon 4$ on $A \beta$ in LOAD was dose dependent, meaning that patients with two alleles had lower levels of $A \beta$ in the CSF than those with one allele [20]. The results of Mehrabian et al. are similar to our results. This may indicate that although APOE $\varepsilon 4$ is of importance for the development of LOAD, other factors may be of greater importance among younger patients [14].

The association between APOE $\varepsilon 4$ status and $A \beta$, and the lack of associations between APOE $\varepsilon 4$ status and the tau proteins was also reported by Price et al., who conducted a postmortem analysis of cognitively intact old people [13]. His results may be a result of aging, or it may be that many of the people examined postmortem had preclinical $\mathrm{AD}$, which could have been expressed as clinical $\mathrm{AD}$ later, as found in a study by Monge-Argiles et al. [34]. In that study where patients with MCI and healthy controls were included, a higher conversion rate from $\mathrm{MCI}$ to $\mathrm{AD}$ was shown among the oldest patients (> 74 years old) and in APOE $\varepsilon 4$ carriers regardless of age [34]. In our study, the association between the APOE genotype and decreased levels of $A \beta$ was found only in the two oldest patient groups of $\mathrm{AD} / \mathrm{aMCI}$ (65 years and older).

However, the increasing effect of APOE $\varepsilon 4$ on A $\beta$ but not on tau pathology with advanced age is found in other studies among people with normal cognition as well [17]. In a previous study by Morris et al., the role of APOE genotype in with advancing age in people without cognitive impairment was explored [17]. They found that both age and APOE $\varepsilon 4$ were associated with lower levels of $A \beta$ in older people with normal cognition, and that the deposition of amyloid plaques starts in middle age and increases throughout life $34.2 \%$ in the age group $70-79$ years, increasing to $50 \%$ in the age group $80-$ 89 years, who had reduced levels of $A \beta$ ). The effect of age was independent of the presence of APOE $\varepsilon 4$ allele [17]. These results are similar to the results in the Price et al. study [13]. In our study there was a significant association between age and $A \beta$ among $A D$ and $\mathrm{aMCI}$ patients who had none or one APOE $\varepsilon 4$ allele, but not in the patients with two alleles who had lower levels of $A \beta$ in all three age groups; see Table 2 . These results are not reflected in the recommended cutoffs of the CSF markers used in clinical practice as there are age specific cutoffs for T-tau, but not for $A \beta$.

The associations already mentioned were however only valid among the patients with MMSE $\geq 25$ where age influenced the levels of $A \beta$, but not the levels of T-tau or P-tau in the adjusted regression analysis; see Tables 3, 4 and 5. In addition, a weaker association was found between APOE $\varepsilon 4$ and A, P-tau and T-tau among these patients.

In the group of patients with MMSE $<25$, we could not find any association between the APOE $\varepsilon 4$ and $A \beta$ or the tau proteins. In the study by Mehrabian et al., the mean MMSE score was 22.3 (SD: 4.3) which makes it comparable with this group. In their study no differences were found in the CSF levels of $A \beta$ between patients with EOAD vs. LOAD [19], which is in line with our findings.

The women in this study had lower levels of $A \beta$ than the men. However, the women had poorer results on all the cognitive tests, indicating a more severe cognitive impairment in line with the findings of Mehrabian et al. [20]. In the multiple regression analysis, sex was not significant after adjusting for cognition. In a study by Rosen et al., women had lower $\mathrm{A} \beta$ and higher of P-tau and T-tau levels as compared to men independent of the degree of cognitive impairment. In that study, the APOE E genotype was not known [35]. As some studies have found that women having a higher APOE $\varepsilon 4$ associated risk of developing $\mathrm{AD}$ than men, this could have influenced the results of that study [23].

\section{Strengths}

The patients in this study represent the types of patients who are normally referred to our memory clinics and were not selected otherwise. All patients underwent a standardized comprehensive assessment including neuropsychological examination, physical examinations including blood and spinal fluid samples, and supplementary brain imaging.

\section{Limitations}

Although it continues to be emphasized that $\mathrm{AD}$ is a clinical diagnosis, both in the revision of the NINCDSADRDA and in the IWG-2 revision of the research criteria, clinical diagnoses are not always correct $[4,36]$. However, we believe that, in the present study, few patients have been misdiagnosed. Experienced physicians made the diagnoses based upon the comprehensive assessment in order to minimize variations. Another challenge was that in some analyses the groups were too small to achieve significant results. 
A further challenge when using CSF biomarkers is interlaboratory and intralaboratory variations, to which $A \beta$ is especially vulnerable [36]. In this study, the same laboratory equipment was used, and the same laboratory analysed all samples to reduce pre-analytical variability.

\section{Conclusion}

In this study conducted among memory clinic patients, we found that in addition to an $\mathrm{AD}$ diagnosis, $\mathrm{A} \beta$ was associated with age, by the presence of one or two APOE $\varepsilon 4$ alleles, and by the MMSE score. As expected, the levels of T-tau and P-tau were both associated with a diagnosis of $\mathrm{AD}$, the MMSE score, but also with by the presence of one or two APOE $\varepsilon 4$ alleles. These findings were valid for patients with a MMSE score $\geq 25$. Among patients with a MMSE score $<25$, these variables explained very little of the variance, meaning that other factors may be of importance. In clinical practice, age specific cutoffs should be considered not only for T-tau, but also for $A \beta$.

\section{Abbreviations}

AD: Alzheimer's dementia; AHUS: Akershus University Hospital; aMCl: Amnestic mild cognitive impairment; APOE: Apolipoprotein E; A : Amyloid $\beta ;$ CDT: Clock Drawing Test; CERAD: The Consortium to Establish a Registry of Alzheimer's Disease; CSF: Cerebrospinal fluid; DaTscan: Dopamine transporter imaging; DLB: Dementia with Lewy Bodies; EOAD: Early onset Alzheimer's dementia; FDG-PET: 18F-2-fluoro-2-deoxy-D-glucose positron emission tomography; FTD: frontotemporal dementia; ICD-10: International Classification of Diseases; IWG: International Working Group; LOAD: Late onset Alzheimer's dementia; MCl: Mild cognitive impairment; MMSE: Mini-Mental State Examination; MRI: Magnetic Resonance Imaging; NINCDS-ADRDA: National Institute of Neurological and Communicative Disorders and stroke - Alzheimer's Disease and Related Disorders Association; OUH: Oslo University Hospital: PDD: Parkinson Disease Dementia; P-tau: Phospho tau; SD: Standard deviation; SP: Specificity; SPECT: Single-photon emission computer tomography; SS: Sensitivity; TMT A and B: Trail Making Tests A and B; T-tau: Total tau; VaD: Vascular dementia

\section{Acknowledgements}

The authors thank all the patients for participating and the staff at our memory clinics for their assistance in this study. The authors thank the Norwegian Register of Persons assessed for Cognitive Symptoms in Specialist Health Care Services for letting us use the data from the registry.

\section{Funding}

Anne-Brita Knapskog receives funding from the Norwegian Health Association's research program. The institution had no further role in study design, neither in the collection, analysis, nor the interpretation of data, in writing of the report, nor in the decision to submit the paper for publication.

\section{Availability of data and materials}

The dataset generated from the patients assessed at Oslo University Hospital, Ullevaal, are available in "The Norwegian Register of Persons assessed for Cognitive Symptoms in Specialist Health Care Services" (NorCog), but restrictions apply to the availability of these data, which were used under license for the current study, and so are not publicly available. Data from both groups of patients are however available from the authors upon reasonable request and with permission of Norwegian National Advisory Unit on Ageing and Health (Ageing and Health).

\section{Authors' contributions}

KE and $A B K$ designed the study. ABK, RSE, $A B$ and IS contributed to the assessment of the patients, and all authors contributed in diagnosing the patients. ABK, KE and IS analysed the data, and all authors interpreted the results. All authors contributed in writing the manuscript, and also in revising the manuscript. All authors read and approved the final manuscript. All authors agreed to be accountable for all aspects of this work. All questions related to the accuracy and integrity of this work are appropriately investigated and resolved.

\section{Ethics approval and consent to participate}

The patients and their family caregivers gave their written consent. The Regional Ethics Committee for medical research in the Southeast of Norway and the Norwegian Data Inspectorate approved the study (reference number 2012/841).

\section{Consent for publication}

Not applicable.

\section{Competing interests}

The authors declare that they have no competing interests.

\section{Publisher's Note}

Springer Nature remains neutral with regard to jurisdictional claims in published maps and institutional affiliations.

\section{Author details}

${ }^{1}$ Department of Geriatric Medicine, The memory clinic, Oslo University Hospital, Ullevaal, Postboks 4956, Nydalen, 0424 Oslo, Norway. ²Department of Neuromedicine and Movement Science, Norwegian University of Science and Technology (NTNU), Trondheim, Norway. ${ }^{3}$ Norwegian National Advisory Unit on Ageing and Health, Vestfold Hospital Trust, Postboks 2136, 3103 Tønsberg, Norway. ${ }^{4}$ Department of Geriatrics, St. Olav Hospital, University Hospital of Trondheim, Trondheim, Norway.

Received: 18 May 2017 Accepted: 6 September 2017

Published online: 11 September 2017

\section{References}

1. Ferri CP, Prince M, Brayne C, Brodaty H, Fratiglioni L, Ganguli M, et al. Global prevalence of dementia: a Delphi consensus study. Lancet. 2005;366(9503): 2112-7

2. Prince $M$, Bryce $R$, Albanese E, Wimo A, Ribeiro W, Ferri CP. The global prevalence of dementia: a systematic review and metaanalysis. Alzheimers Dement J Alzheimers Assoc. 2013;9(1):63-75. e62

3. World Health Organization. http://www.who.int/mediacentre/factsheets/ fs362/en/ Assessed 14 Aug 2017

4. McKhann GM, Knopman DS, Chertkow H, Hyman BT, Jack CR Jr, Kawas CH, et al. The diagnosis of dementia due to Alzheimer's disease: recommendations from the National Institute on Aging-Alzheimer's Association workgroups on diagnostic guidelines for Alzheimer's disease. Alzheimers Dement J Alzheimers Assoc. 2011;7(3):263-9.

5. Hardy JA, Higgins GA. Alzheimer's Disease: the amyloid cascade hypothesis. Science. 1992;256(5054):184-5.

6. Braak H, Zetterberg H, Del Tredici K, Blennow K. Intraneuronal tau aggregation precedes diffuse plaque deposition, but amyloid-beta changes occur before increases of tau in cerebrospinal fluid. Acta Neuropathol. 2013;126(5):631-41.

7. Mattsson N, Blennow K, Zetterberg H. CSF biomarkers: pinpointing Alzheimer pathogenesis. Ann N Y Acad Sci. 2009;1180:28-35.

8. Slaets S, Le Bastard N, Theuns J, Sleegers K, Verstraeten A, De Leenheir E, et al. Amyloid pathology influences abeta1-42 cerebrospinal fluid levels in dementia with lewy bodies. J Alzheimers Dis. 2013:35(1):137-46.

9. Jansen WJ, Ossenkoppele R, Knol DL, Tijms BM, Scheltens P, Verhey FR, et al. Prevalence of cerebral amyloid pathology in persons without dementia: a meta-analysis. JAMA J Am Med Assoc. 2015;313(19):1924-38.

10. Jack CR Jr, Wiste HJ, Weigand SD, Rocca WA, Knopman DS, Mielke MM, et al. Age-specific population frequencies of cerebral beta-amyloidosis and neurodegeneration among people with normal cognitive function aged 5089 years: a cross-sectional study. Lancet Neurol. 2014:13(10):997-1005.

11. Blennow K, Hampel H, Zetterberg H. Biomarkers in amyloid-beta immunotherapy trials in Alzheimer's disease. Neuropsychopharmacol Officia Publ Am Coll Neuropsychopharmacol. 2014;39(1):189-201.

12. Braak H, Braak E. Frequency of stages of Alzheimer-related lesions in different age categories. Neurobiol Aging. 1997;18(4):351-7. 
13. Price JL, McKeel DW Jr, Buckles VD, Roe CM, Xiong C, Grundman M, et al. Neuropathology of nondemented aging: presumptive evidence for preclinical Alzheimer disease. Neurobiol Aging. 2009;30(7):1026-36.

14. Yu JT, Tan L, Hardy J. Apolipoprotein E in Alzheimer's disease: an update. Annu Rev Neurosci. 2014;37:79-100.

15. Huang Y. Abeta-independent roles of apolipoprotein E4 in the pathogenesis of Alzheimer's disease. Trends Mol Med. 2010;16(6):287-94.

16. Hardy J, Bogdanovic N, Winblad B, Portelius E, Andreasen N, CedazoMinguez A, et al. Pathways to Alzheimer's disease. J Intern Med. 2014;275(3): 296-303.

17. Morris JC, Roe CM, Xiong C, Fagan AM, Goate AM, Holtzman DM, et al. APOE predicts amyloid-beta but not tau Alzheimer pathology in cognitively normal aging. Ann Neurol. 2010;67(1):122-31.

18. Lautner R, Palmqvist S, Mattsson N, Andreasson U, Wallin A, Palsson E, et al. Apolipoprotein E genotype and the diagnostic accuracy of cerebrospinal fluid biomarkers for Alzheimer disease. JAMA Psychiatry. 2014;71(10):1183-91.

19. Vemuri P, Wiste HJ, Weigand SD, Knopman DS, Shaw LM, Trojanowski JQ, et al. Effect of apolipoprotein E on biomarkers of amyloid load and neuronal pathology in Alzheimer disease. Ann Neurol. 2010;67(3):308-16.

20. Mehrabian S, Alexopoulos P, Ortner M, Traykov L, Grimmer T, Kurz A, et al. Cerebrospinal fluid biomarkers for Alzheimer's disease: the role of apolipoprotein E genotype, age, and sex. Neuropsychiatr Dis Treat. 2015;11:3105-10.

21. Scheltens P, Blennow K, Breteler MM, de Strooper B, Frisoni GB, Salloway S, et al. Alzheimer's Disease. Lancet. 2016;388(10043):505-17.

22. Eisenberg DT, Kuzawa CW, Hayes MG. Worldwide allele frequencies of the human apolipoprotein E gene: climate, local adaptations, and evolutionary history. Am J Phys Anthropol. 2010;143(1):100-11.

23. Genin E, Hannequin D, Wallon D, Sleegers K, Hiltunen M, Combarros $\mathrm{O}$, et al. APOE and Alzheimer disease: a major gene with semi-dominant inheritance. Mol Psychiatry. 2011;16(9):903-7.

24. Blennow K, Hampel H. CSF markers for incipient Alzheimer's disease. Lancet Neurol. 2003:2(10):605-13.

25. Knapskog AB, Engedal K, Braekhus A. Performance of cerebrospinal fluid biomarkers of Alzheimer disease in a memory Clinic in Norway. Alzheimer Dis Assoc Disord. 2016;30(1):8-14

26. Mattsson N, Zetterberg H, Hansson O, Andreasen N, Parnetti L, Jonsson M, et al. CSF biomarkers and incipient Alzheimer disease in patients with mild cognitive impairment. JAMA. 2009;302(4):385-93.

27. Braekhus A, Ulstein I, Wyller TB, Engedal K. The memory clinic-outpatient assessment when dementia is suspected. Tidsskr Nor Laegeforen. 2011;131(22):2254-7.

28. Alzheimer's Association QC Program for CSF biomarkers. http://www.alz.org/ research/funding/global_biomarker_projects.asp\#tstandardization. Accessed 10 Jan 2017.

29. Winblad B, Palmer K, Kivipelto M, Jelic V, Fratiglioni L, Wahlund LO, et al. Mild cognitive impairment-beyond controversies, towards a consensus: report of the international working group on mild cognitive impairment. Intern Med. 2004;256(3):240-6.

30. World Health Organization. The ICD-10 Classification of Mental and Behavioural Disorders: Diagnostic Criteria for Research. Geneva: World Health Organization; 1993.

31. Neary D, Snowden JS, Gustafson L, Passant U, Stuss D, Black S, et al. Frontotemporal lobar degeneration: a consensus on clinical diagnostic criteria. Neurology. 1998;51 (6):1546-54.

32. McKeith IG, Dickson DW, Lowe J, Emre M, O'Brien JT, Feldman H, et al. Diagnosis and management of dementia with Lewy bodies: third report of the DLB consortium. Neurology. 2005;65(12):1863-72.

33. Sando SB, Melquist S, Cannon A, Hutton ML, Sletvold O, Saltvedt I, et al. APOE epsilon 4 lowers age at onset and is a high risk factor for Alzheimer's disease; a case control study from central Norway. BMC Neurol. 2008;8:9.

34. Monge-Argiles JA, Gasparini-Berenguer R, Gutierrez-Agullo M, Munoz-Ruiz C, Sanchez-Paya J, Leiva-Santana C. Influence of APOE genotype on Alzheimer's disease CSF biomarkers in a Spanish population. Biomed Res Int 2016;2016:1390620.

35. Rosen C, Farahmand B, Skillback T, Nagga K, Mattsson N, Kilander L, et al. Benchmarking biomarker-based criteria for Alzheimer's disease: data from the Swedish dementia registry, SveDem. Alzheimers Dement J Alzheimers Assoc. 2015;11(12):1470-9.

36. Dubois B, Feldman HH, Jacova C, Hampel H, Molinuevo JL, Blennow K, et al. Advancing research diagnostic criteria for Alzheimer's disease: the IWG-2 criteria. Lancet Neurol. 2014;13(6):614-29.

\section{Submit your next manuscript to BioMed Central and we will help you at every step:}

- We accept pre-submission inquiries

- Our selector tool helps you to find the most relevant journal

- We provide round the clock customer support

- Convenient online submission

- Thorough peer review

- Inclusion in PubMed and all major indexing services

- Maximum visibility for your research

Submit your manuscript at www.biomedcentral.com/submit
Biomed Central 